\title{
Food futures: Storylines of dietary megatrends along the Shared Socioeconomic Pathways (SSPs)
}

\author{
$\underline{\text { Roberta Alessandrini }}^{1}$ and Benjamin Leon Bodirsky ${ }^{2}$ \\ ${ }^{1}$ Wolfson Institute of Preventive Medicine, Queen Mary University of London, London, United Kingdom and \\ ${ }^{2}$ Potsdam Institute for Climate Impact Research (PIK), Potsdam, Germany
}

\section{Abstract}

Under- and over-nutrition are among the most important global health determinants, and the agro-food system is the main interface between human society and the natural world and thereby responsible for land cover change, water withdrawals, greenhouse gases, biodiversity loss and nutrient pollution.

We formulated five qualitative storylines of future dietary developments using the Shared Socioeconomic Pathways (SSPs), a tool widely used in the climate change research community. Our storylines diverge in respect to the main actors in the food system, the policy framework, the food supply chain organisation, consumer context, dietary composition, health systems and health outcomes in respect to under- and over-nutrition. We titled each storyline with combinations of iconic foods that would be representative of each future.

SSP1 = Minestrone and Enjera (sustainable diets). The civil society is the main actor in shaping the food system and diets are mainly unprocessed and plant-based. The health burden from under- and overnutrition is drastically reduced in societies all over the world.

SSP2 = Pizza Hawaii with a side salad and banana (middle of the plate). This is a continuation of the current trends. The food industry is the main actor of the food system providing highly marketed processed food. Undernutrition slowly declines in the least developed countries, but obesity and NCDs increase drastically in both LMIC and HIC.

SSP3 = Kohlroulade and instant noodles (rocky road). Populistic and monopolistic governments are the main actors in shaping the food system. Diets are made of traditional and unhealthy dishes. Obesity and micronutrient deficiencies coexist and the nutritionrelated burden of disease is high.

SSP4 = Kaviar and beans (inequality). A small and wealthy elite is the key actor shaping the political and the global food system. Diets are luxurious, highly customised and marketed for wealthy, and consisting of cheap and simple ingredients for the vast majority. While the elite enjoys healthy and long lives, malnutrition and stunting remain widespread in LMICs; obesity and diet-related NCDs decline everywhere.

SSP5 = Artificial meat burger with a skinny milkshake (fossil fuel). A few large tech companies are the main actors shaping the food system. Algorithms based on big-data provide customised diets consisting of superfoods and new foods. Under-nutrition disappears, BMI converges to normal levels, while diet-related NCDs remain prevalent.

The existing storylines in the literature do not focus on the agro-food system and on health and nutrition outcomes. Our work contributes to the ongoing conversation on food futures.

\section{Conflict of Interest}

There is no conflict of interest 